\title{
Flow profiling of a surface-acoustic-wave nanopump
}

\author{
Z. Guttenberg, ${ }^{1}$ A. Rathgeber, ${ }^{1}$ S. Keller, ${ }^{2}$ J. O. Rädler, ${ }^{2}$ A. Wixforth, ${ }^{3}$ M. Kostur, ${ }^{3}$ M. Schindler, ${ }^{3}$ and P. Talkner ${ }^{3}$ \\ ${ }^{1}$ Advalytix AG, Eugen-Sänger-Ring 4, D-85649 Brunnthal, Germany \\ ${ }^{2}$ Ludwig-Maximilians-Universität, Geschwister-Scholl-Platz 1, D-80539 München, Germany \\ ${ }^{3}$ Institut für Physik, Universität Augsburg, Universitätsstrasse 1, D-86135 Augsburg, Germany \\ (Received 6 May 2004; revised manuscript received 16 August 2004; published 23 November 2004)
}

\begin{abstract}
The flow profile in a capillary gap and the pumping efficiency of an acoustic micropump employing surface acoustic waves is investigated both experimentally and theoretically. Ultrasonic surface waves on a piezoelectric substrate strongly couple to a thin liquid layer and generate a quadrupolar streaming pattern within the fluid. We use fluorescence correlation spectroscopy and fluorescence microscopy as complementary tools to investigate the resulting flow profile. The velocity was found to depend on the applied power approximately linearly and to decrease with the inverse third power of the distance from the ultrasound generator on the chip. The found properties reveal acoustic streaming as a promising tool for the controlled agitation during microarray hybridization.
\end{abstract}

DOI: 10.1103/PhysRevE.70.056311

PACS number(s): 47.15.Gf, 47.85.Np, 83.50.Xa, 83.10.Pp

\section{INTRODUCTION}

DNA hybridization technology has undergone a rapid development in the past years. In particular, the advent of DNA microarrays (DNA chips) has revolutionized gene expression analysis. Yet the accuracy and reproducibility of the hybridization assays are often limited by microfluidic constraints. The main principle of a DNA array is a glass slide with small probe spots of single-stranded DNA covalently bound to the surface. A solution containing fluorescently labeled sample molecules is then spread across the microarray where the molecules are allowed to bind to the respective probe spots. Successful hybridization is usually observed as an increase in the fluorescence intensity on a spot. Usually, such experiments are performed in sealed chambers with a typical film thickness of less than 100 microns. Thin liquid films exhibit very small Reynolds numbers, and the molecules in the fluid move predominantly diffusively [1]. Diffusion, however, is a very slow process. Even for small molecules such as rhodamine, the distance traveled in water after $10^{3} \mathrm{~s}$ is only about $1 \mathrm{~mm}$. For relatively large DNA molecules, the diffusion-driven displacement will be even smaller. Hence, typical hybridization assays are performed overnight or even over a weekend, and then are stopped at some more or less arbitrary point. Such experiments are also referred to as "end point" experiments, but one cannot be sure whether the equilibrium of the reaction is reached for every spot. However, if the DNA binding reactions are not in equilibrium at the end of the hybridization time, the results can be misleading. Quite obviously, this process can be accelerated if the molecules were to be actively transported along the solid surface plane. As the amount of DNA available for a hybridization experiment is often very limited, one has to reduce the volume as much as possible. Therefore, pump-driven flow systems [2] usually having large dead volumes are not convenient to induce the molecule transport during the hybridization process. There are numerous other possibilities to actuate thin fluid films, among them, for example, electrokinetic forces $[3,4]$.
In this work, we describe a technique to actuate a thin liquid film in a capillary gap, report on measurements of the hydrodynamic flow field in such a gap, and compare to theoretical calculations. We employ surface acoustic waves (SAW) propagating on the surface of a piezoelectric solid to induce acoustic streaming and mixing within the fluid. To understand the fluid dynamics in such a system, we concentrate on how the acoustic streaming transforms into a velocity field profile within a homogeneous DNA solution in the capillary gap. A SAW can be excited by the application of a radio frequency signal (rf) to an interdigital transducer (IDT) on a piezoelectric substrate. The wavelength of the excited SAW is defined by the geometry of the IDT. Typical displacement amplitudes of a SAW are in the nanometer range, depending on the applied rf power. If the SAW supporting surface of the substrate is in contact with a liquid, and if the SAW has any displacement component normal to the surface, ultrasonic SAW power leaks into the fluid in the form of an acoustic wave. This so-called leaky SAW (LSAW) is expected to decay exponentially with distance from its source.

The interaction between the SAW and the liquid film leads to an internal, acoustically induced streaming. The reason for this is a net pressure gradient in the direction of the sound propagation in the fluid. Usually, this leads to the generation of a fluidic jet, which can be nicely visualized employing a dye solution and a fluorescence video microscope. The captured images are evaluated in a subsequent imageprocessing step, being a versatile tool for the interpretation of dynamic processes. This method yields real images of the observed sample as a whole, and therefore reduces the problem of a false interpretation of the data. For more precise measurement of the flow field and velocity, we use fluorescence correlation spectroscopy (FCS) [5]. In this method, the movement of fluorescent particles can be determined in the focal spot of a confocal microscope by evaluating the time autocorrelation function of the intensity fluctuations. Successive measurements at different positions inside the fluidic device yield a velocity map. 


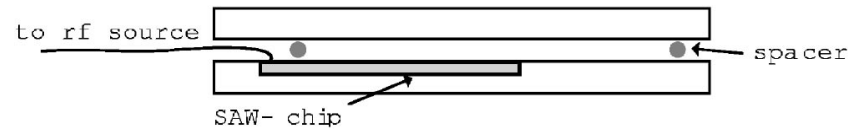

FIG. 1. The measurement setup for the evolution of dye spots consists of a $200 \mu \mathrm{m}$ slit between two plexiglas plates (3 $\times 3 \mathrm{~cm}^{2}$ ) kept in the right distance with spacers. Integrated in the lower plate is the SAW chip, being connected to the rf source outside the fluid film. The transducer is located in the middle of the plate. Images were taken with a video microscope from above through the upper plate.

\section{THE EXPERIMENTAL SETUP AND MICROFLUIDIC CHARACTERISTICS}

We assembled a special microfluidic arrangement to induce streaming in a narrow capillary gap between a glas slide and a plexiglass block, containing a SAW device.

Figure 1 gives a sketch of the principal setup as seen from the side. Figure 2 shows the chip architecture. The width of the gap is controlled by spacers; it can be continuously adjusted down to $30 \mu \mathrm{m}$. In the measurements described, we worked with a spacer of $200 \mu \mathrm{m}$. The SAW chip consists of a $128^{\circ}$ rotated Y-cut, $X$ propagating $\mathrm{LiNbO}_{3}$ single crystal chip.

The IDT consists of 22 finger pairs, $0.85 \mathrm{~mm}$ aperture, and a period $\lambda=28 \mu \mathrm{m}$. The IDT fingers are thermally evaporated 300-nm-thick Au electrodes, fabricated by a standard photolithographic technique. The complete chip was protected with an 800-nm-thick, sputtered silicon dioxide layer, which was removed at the contact pads to allow for electrical connection. The IDT was driven with an ac signal at the resonance frequency $f_{0}$, being defined as $f_{0}=c / \lambda$. For a SAW velocity $c \approx 3800 \mathrm{~m} / \mathrm{s}$, the frequency $f_{0}$ is hence about $136 \mathrm{MHz}$. Such an IDT efficiently converts the applied rf signal into an acoustic wave, which in this case is launched as a bidirectional beam perpendicular to the fingers.

If the capillary gap is filled with a fluid, switching on the rf power leads to a flow pattern as sketched in Fig. 3.

The fluid is expelled from the transducer region to the left and right side and flows back to the IDT from bottom and top. According to the high viscous damping, the flow velocity decreases rapidly with distance from the IDT.

Further investigations were focused on the characterization of the flow field. The issue of this work was to find out how the setup can be optimized for agitating a large hybridization area. For this purpose, we first want to discuss the nature of the observed SAW-induced motion.

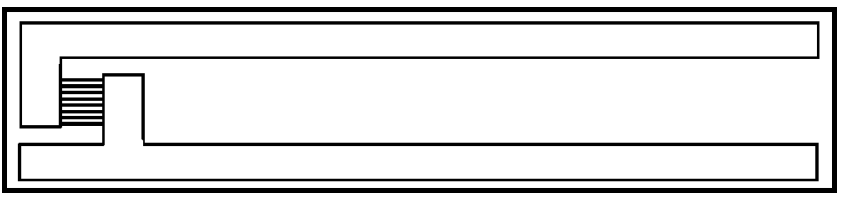

FIG. 2. The graph shows the sketch of the $4 \times 23 \mathrm{~mm}^{2} \mathrm{LiNbO}_{3}$ chip with gold structures. Situated on the left side is the interdigitated transducer $\left(0.85 \times 1 \mathrm{~mm}^{2}\right)$ inducing the SAW perpendicular to the long side of the chip. The contact wires are connected with the rf power source on the right end.

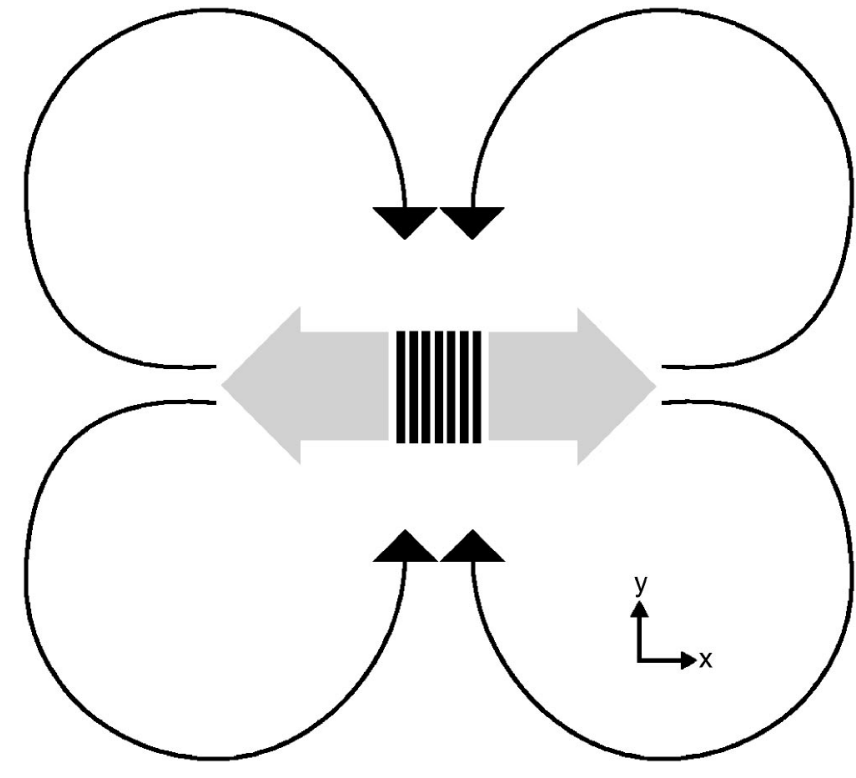

FIG. 3. Flow pattern generated by SAW. The fluid is pumped perpendicular to the metal fingers of the IDT and is sucked in parallel to them.

The propagating LSAW couples to a sound wave in the liquid. Because the sound velocity in fluids $c_{\text {liquid }}$ is always smaller than the LSAW velocity in the solid substrate, sound is radiated into the liquid under an angle obeying Snellius' law of diffraction,

$$
\sin \left(\theta_{R}\right)=\frac{c_{\text {liquid }}}{c_{\text {LSAW }}} .
$$

This can be understood as a consequence of phase matching between the LSAW and the radiated sound beam in the liquid. This "mode conversion" leads to an exponential decay of the LSAW. In our case, the observed damping in power was about $1 \mathrm{dBm}$ per wavelength, which is in good accordance with values found in Refs. [6] and [7]. This results in a decrease of $35 \mathrm{dBm}$ per $\mathrm{mm}$. In the following, we will give a short overview of how the sound radiated in the fluid can induce a streaming force.

Sound traveling through a liquid is attenuated by the viscosity along its transmission through the medium. If the intensity is high, this attenuation creates an acoustic pressure gradient along the propagation of the wave. The gradient induces a force in the same direction that causes a flow in the fluid. This conversion of an attenuated sound wave into a steady flow is a nonlinear effect which is known as acoustic streaming. When a SAW, i.e., a surface acoustic wave with a spatially constant amplitude, is in contact with a fluid, the acoustic streaming is only a minor effect [6]. Much stronger is the streaming that results from the exponential decay of the LSAW. This decay causes the conversion into sound waves in the liquid within a short distance, leading to a large gradient of the sound amplitude and consequently to a strong force that generates the acoustic streaming. Acoustic streaming has been described in detail in Refs. [8,9]. In short, the acoustic wave excerts an effective force on the fluid that can 


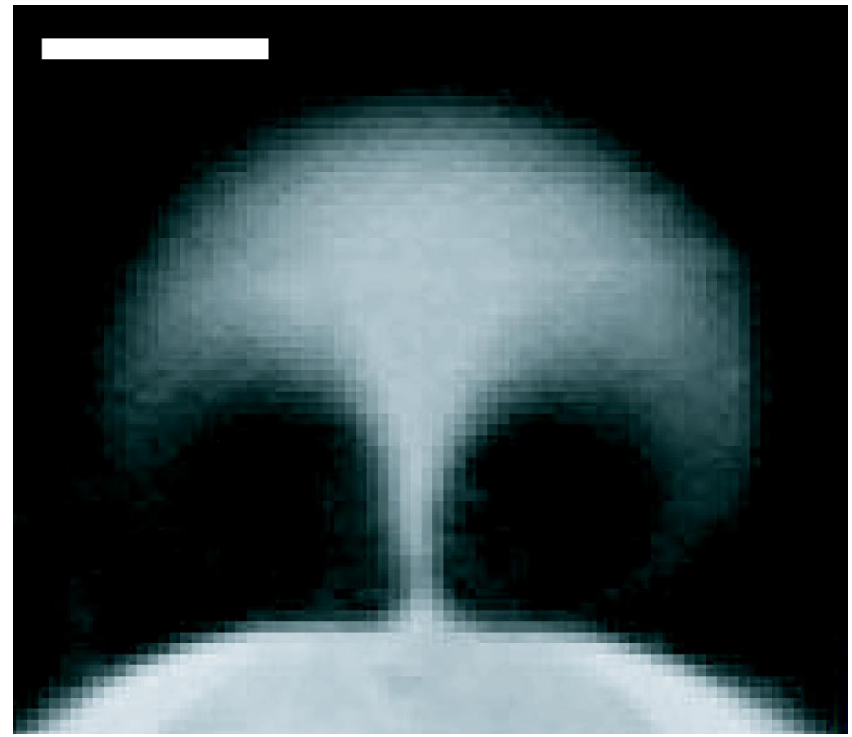

FIG. 4. A dye jet pumped by the SAW transducer, shortly after switching the rf power. In the middle of the transducer, the fluid is pumped upwards; on both sides the fluid is sucked in. The bar is $0.5 \mathrm{~mm}$ wide.

be expressed in terms of the sound velocity field in the fluid [8],

$$
\mathbf{F}=\rho\left\langle\left(\mathbf{v}_{f} \cdot \boldsymbol{\nabla}\right) \mathbf{v}_{f}+\mathbf{v}_{f}\left(\boldsymbol{\nabla} \cdot \mathbf{v}_{f}\right)\right\rangle,
$$

where the angular brackets denote a time average that removes the fast oscillations of the sound wave.

Finally, we note that the force is proportional to $f_{0}^{2}$. High force densities, therefore, can be achieved by means of high LSAW frequencies. On the other hand, lower frequencies lead to smaller damping rates of the LSAW and the force density acts on a larger volume. For an optimal design, a trade-off has to be found.

\section{MATERIALS AND METHODS}

\section{A. Fluorescence microscopy}

\section{Microscope}

To visualize the activity of the SAW transducer, a NaFluorescein solution $(1 \mathrm{mg} / \mathrm{mL})$ is pipetted into a small hole in the cover of the fluid film. For the excitation of the fluorescence of the dye, blue LEDs are attached to a ring some distance above the cover. Fluorescence images are taken by a video microscope (Olympus, Hamburg) with a suitable filter to suppress the excitation light.

\section{Image processing}

Fluorescence images of the evolving Na-Fluorescein spot (see Figs. 4 and 5) were taken every $10 \mathrm{~s}$ and stored into an avi movie file. The avi movie was further processed by a procedure converting the pictures into binary images with the help of the Shannon entropy function and detecting the edges of the dye spot with the particle finder algorithm. The coordinates of every edge line were put together in one file

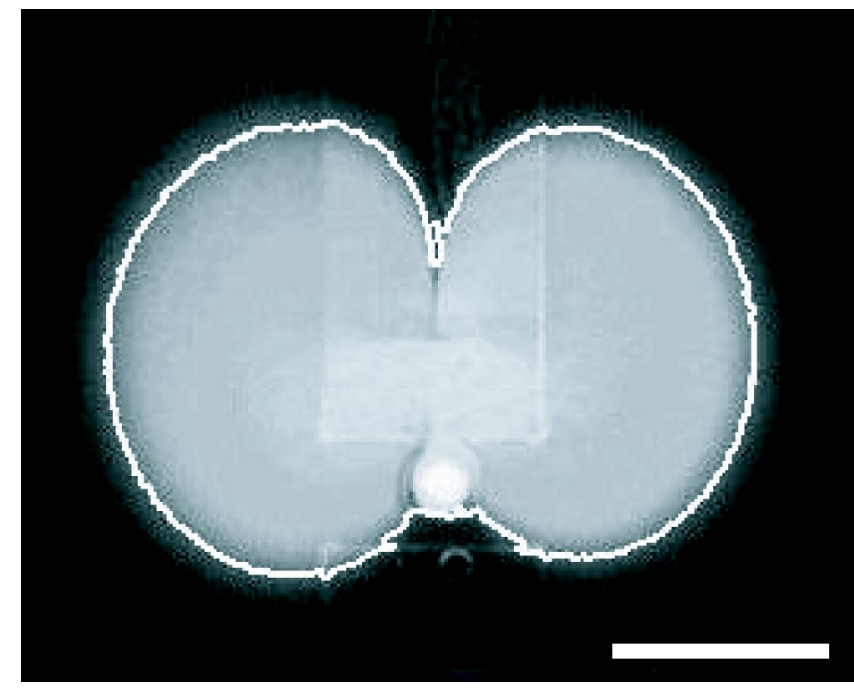

FIG. 5. A dye spot after $5 \mathrm{~min}$ of SAW pumping $(40 \mathrm{~mW})$ is shown. Superimposed is the edge line calculated by the image procedure. The bar is $1 \mathrm{~mm}$ wide.

(Fig. 6) and the positions of the leading edge to the left and the right were extracted for every recorded point of time. This leads to a distance versus time diagram (Fig. 7). Differentiating these data yields a speed versus distance diagram. Also, the area of the Na-Fluorescein spot and the speed of the area increase can be calculated.

\section{B. Fluorescence correlation spectroscopy}

The raw signal in FCS [10-13] experiments is the timedependent intensity signal coming from a small and fixed volume $(\approx 0.5 \mathrm{fl})$ created by a laser focus. Fluorescently labeled molecules entering and leaving the open volume lead to fluctuations in the recorded signal $F(t)$,

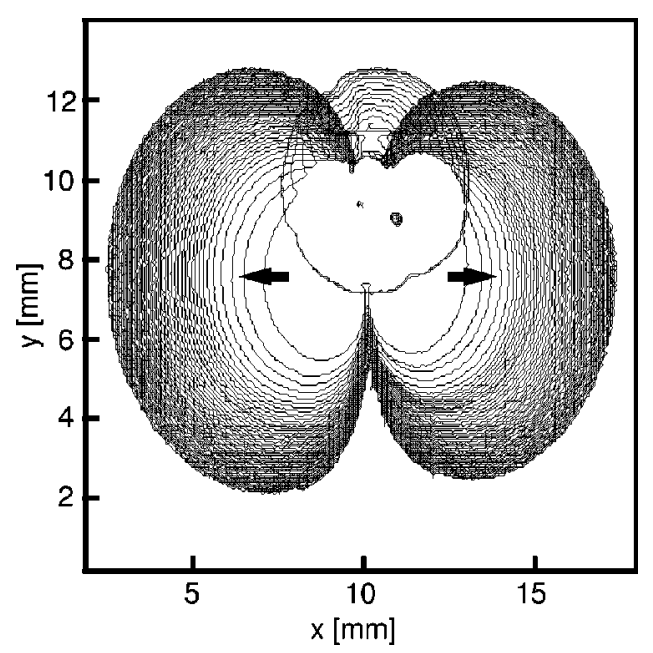

FIG. 6. The edges of a dye spot during the pumping of the SAW transducer $(40 \mathrm{~mW})$ are recorded every $10 \mathrm{~s}$ and put together in one graph. The evolution from a round spot to a butterfly shape can be observed. 


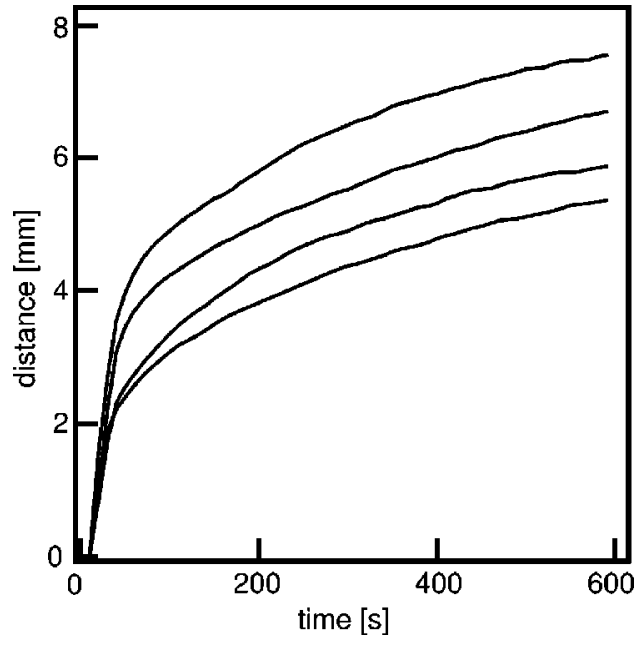

FIG. 7. The distance of the dye front as a function of time for $10,25,63$, and $158 \mathrm{~mW}$ rf power (from bottom to top). The data points were determined every $10 \mathrm{~s}$ at the line, marked by the arrows in Fig. 6 and connected by lines.

$$
F(t)=q Q \int_{V} I(\vec{r}) C(\vec{r}, t) d V .
$$

Here, $q$ stands for constant intensity losses due to the experimental setup, e.g., detector efficiency, filters. $Q$ is the fluorescence quantum yield of the dye and $C(\vec{r}, t)$ denotes the concentration of the labeled particles; $I(\vec{r})=I_{0} \exp \left[-2\left(x^{2}\right.\right.$ $\left.\left.+y^{2}\right) / r_{0}^{2}\right] \exp \left(-2 z^{2} / z_{0}^{2}\right)$ is a combination of the collection efficiency and the excitation profile. If the confocal detection geometry is chosen properly, a Gaussian effective volume with radius $r_{0}$ and height $z_{0}$ is a good approximation [14].

In this work, we want to focus on the possibility to measure flow velocities, as was shown by Gösch et al. [15]. The experimental autocorrelation function $g_{2}(t)$ is calculated from the measured fluorescence intensity $F(t)$ by

$$
g_{2}(t)=\frac{\left\langle F\left(t_{0}\right) F\left(t_{0}+t\right)\right\rangle}{\left\langle F\left(t_{0}\right)\right\rangle^{2}}=1+\frac{\left\langle\delta F\left(t_{0}\right) \delta F\left(t_{0}+t\right)\right\rangle}{\left\langle F\left(t_{0}\right)\right\rangle^{2}} .
$$

In order to obtain the flow velocities without perturbation by diffusion, we used fluorescent microspheres of $1 \mu \mathrm{m}$ radius (FluoSpheres yellow-green 505/515, F-8823, Molecular Probes, Eugene, OR). On the experimentally relevant time scale, the Brownian motion of these spheres is of minor importance [16]. The autocorrelation function $g_{2}(t)$ is then completely determined by the flow

$$
g_{2}(t)=1+\frac{1}{N} G_{\text {flow }}(t)
$$

where

$$
G_{\text {flow }}(t)=\exp \left[-\left(\frac{t}{\tau_{\mathrm{F}}}\right)^{2}\right]
$$

Here, $\tau_{\mathrm{F}}$ is the dwell time of the particles in the focus. For the situation in our experiment, where the radius $R$ of the particles is comparable to or larger than the radius of the observation volume $r_{0}$, the dwell time is given by

$$
\tau_{\mathrm{F}}=\frac{r_{0}+R}{v_{\text {flow }}} .
$$

We used a commercial setup by Carl Zeiss (Jena, Germany) consisting of the module Confocor 2 and the microscope model Axiovert 200 with a Zeiss C-Apochromat $40 \times$ NA 1.2 water immersion objective. For excitation, the $514 \mathrm{~nm}$ line of a $25 \mathrm{~mW}$ argon laser was used. Emitted fluorescence was detected at wavelengths longer than $530 \mathrm{~nm}$ by means of an avalanche photodiode allowing for singlephoton counting.

\section{RESULTS}

As a test for the image processing, a spot of Fluorescein pipetted into the hole of the cover plate was observed for 10 min, with the SAW switched off. The area covered by the dye, therefore, only increases by diffusion. Using the diffusion equation $\left\langle x^{2}\right\rangle=2 D t$, we determined a value of $D$ $=370 \mu \mathrm{m}^{2} / \mathrm{s}$ for the diffusion constant of the dye molecules which is in good agreement with literature values of $200-400 \mu \mathrm{m}^{2} / \mathrm{s}$.

In Fig. 4, a close-up image of the area near the transducer is shown, shortly after starting the SAW. The 1-mm-wide IDT is hidden by the dye solution on the lower side pumping a $200-\mu \mathrm{m}$-wide jet to the upper side. The jet becomes broader with increasing distance and a part is transported to the influx areas of the IDT perpendicular to the direction of pumping. After $10 \mathrm{~min}$, the Fluorescein covered area shows a butterfly shape. Employing the image processing the edge of the dye spot is determined every $10 \mathrm{~s}$ during the observation time. A border line between the colored and uncolored areas computed this way is plotted in Fig. 5. Collecting the traces of a $10 \mathrm{~min}$ experiment into one graph yields a plot as in Fig. 6. The initial dye spot and the succeeding evolution of the edge lines are visible in this graph. These data were used for determining the growth rate of the area (Fig. 8). The growth of a Fluorescein spot was observed for seven different rf powers and the actual position of the front line in the direction of the arrows in Fig. 6 was recorded. The area growth rate decreases rapidly with time until the diffusion of the dye dominates with a diffusion constant of $370 \mu \mathrm{m}^{2} / \mathrm{s}$. In the experiment, this position $x_{D}^{*}$ is found to depend linearly on the logarithm of the rf power, see Fig. 9.

From the movement of the dye front in the symmetry direction marked by the arrows in Fig. 6, the position can be determined as a function of time (Fig. 7). The curves show a continuously decelerating movement with time. With different rf-power values, no qualitative difference can be observed. Differentiating these data numerically and plotting them against the $x$ position gives the flow speed against time. However, the FCS method leads to more accurate values for the flow speed as a function of the lateral position.

Figure 10 shows FCS correlation data taken as a function of the applied power. Equation (5) was used as a model function to fit the data [16]. The small deviations may be attributed to particle size effects [17] or to the polydispersity of the used beads [18]. The fitted dwell times of the particles show a strong dependence on the applied power. According 


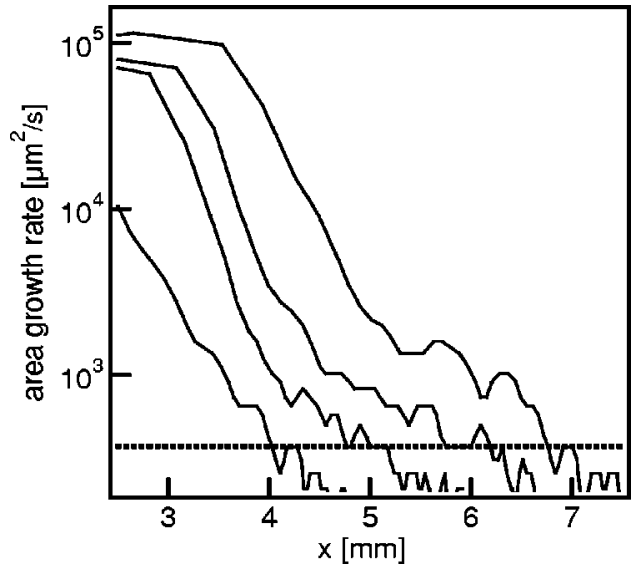

FIG. 8. The area growth rate of the Fluorescein spot is plotted against the particular position of the advancing dye front for four different rf-power values $(10,40,63$, and $158 \mathrm{~mW}$ from left to right). When the speed reaches the region of the diffusion constant of the dye, the data are superposed by noise. The diffusion constant of Fluorescein $\left(370 \mu \mathrm{m}^{2} / \mathrm{s}\right)$ is marked by a dashed line. For small distances, representing early times, the image processing yields misleading results, since the transducer region is covered by the initial round dye spot.

to Eq. (7), the velocity was calculated and plotted as a function of the power (Fig. 11). This was done for three different positions in the channel. The highest velocities were achieved at positions near the chip. The measured data were fitted by a linear dependence of the velocity on the applied power. Flow velocities close to $10 \mu \mathrm{m} / \mathrm{s}$ are not determined correctly (points with large error bars). In Fig. 12, the velocity profile for a selected area close to the chip is shown. The $x$ direction is the direction in which the chip is supposed to pump. To investigate the dependence quantitatively, we took the values indicated by the open squares and plotted their logarithm as a function of the position, see Fig. 13. As can be seen from this graph, the velocity decreases fast with distance from the transducer. Ideally, the velocity at the symme-

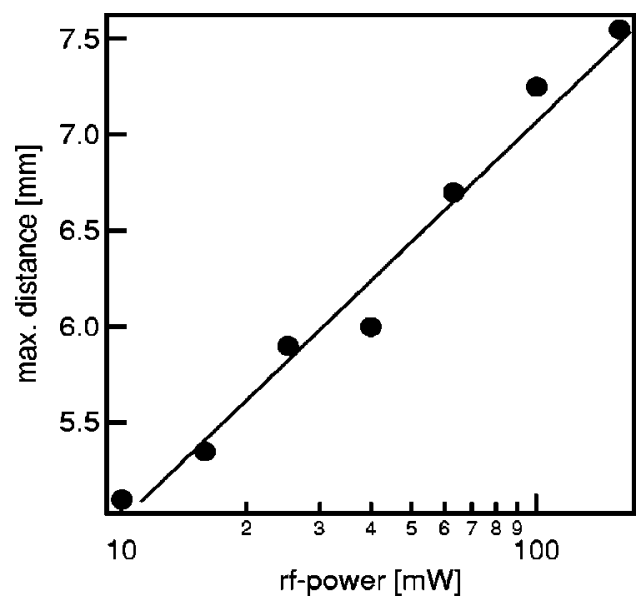

FIG. 9. The distance $x_{D}^{*}$ where the speed of the area growth reaches the diffusion constant of Fluorescein $\left(370 \mu \mathrm{m}^{2} / \mathrm{s}\right)$ depending on the applied rf power. Superimposed to the data is a straight line.

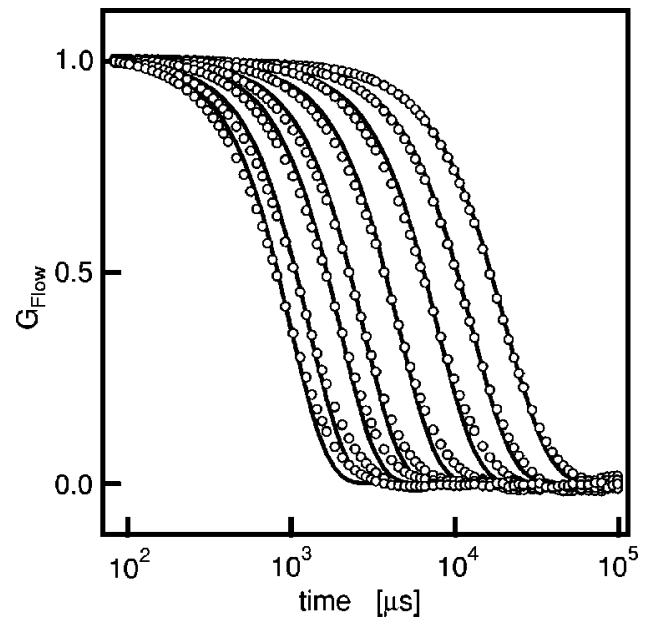

FIG. 10. Normalized autocorrelation curves from FCS measurements. The applied power was varied between $20 \mathrm{~mW}$ (right) and $500 \mathrm{~mW}$ (left) in logarithmic steps. Circles represent the experimental data, whereas the lines are fits to Eq. (6).

try point $x=0$ should vanish. The high gradient of the velocity field there and the experimental positional uncertainty, however, lead to the apparent finite velocity.

\section{THEORY}

\section{A. Calculation of the flow field}

All observed flows in the 200- $\mu$ m-thick fluid film had velocities smaller than $1 \mathrm{~mm} / \mathrm{s}$ and, hence, are characterized by Reynolds numbers smaller than 1 . This means that the flow is completely laminar [19], which simplifies the situation for the calculation of the streamlines. The steady flow is determined by the relatively simple Stokes equations [8],

$$
\mu \Delta \mathbf{v}(\mathbf{x})=\nabla p(\mathbf{x})-\mathbf{F}(\mathbf{x}),
$$

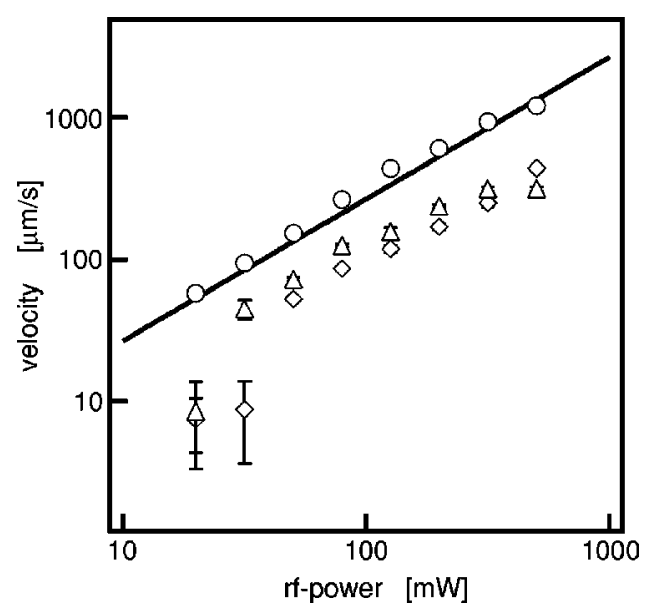

FIG. 11. Flow velocity as a function of the applied power for three different positions indicated by circles, triangles, and squares. The corresponding positions are marked by the same symbols as in Fig. 12. Circles represent the results from the fitted curves in Fig. 10. The line is a fit to the data with a slope of 1 . 


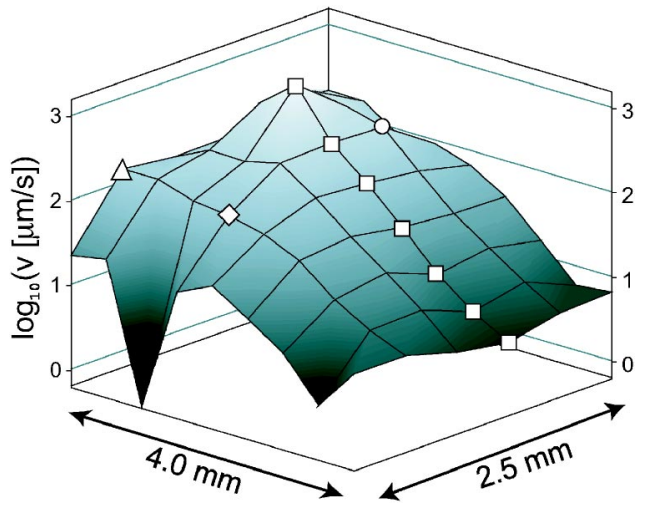

FIG. 12. Velocity profile for a selected area near the pumping chip at a rf power of $79 \mathrm{~mW}$. The triangle, square, and circle mark the positions at which the data shown in Fig. 11 were taken.

$$
\boldsymbol{\nabla} \cdot \mathbf{v}=\mathbf{0},
$$

where $\mu$ denotes the shear viscosity of the fluid. The driving force $\mathbf{F}$ is given in terms of the sound wave by Eq. (2). The SAW induced by the IDT radiates sound waves into the fluid layer that propagate in directions whose lateral components are perpendicular to the IDT fingers. These waves decay rapidly. The strength of the resulting force is largest in regions above the outermost fingers of the IDT and the directions point away from the IDT. In the experiment, the force has dropped by three orders of magnitude on a distance of $1 \mathrm{~mm}$ away from the IDT.

We use these features in order to find an approximate analytical solution of Eqs. (8) and (9) for the time-averaged velocity and pressure fields and simplify the problem by the following assumptions: (i) lateral boundaries are neglected, i.e., we consider an infinitely extended fluid layer of thickness $d$ with no-slip boundary conditions at both confining plates; (ii) we approximate the actual spatially extended force density by a pair of constant forces being concentrated on lines perpendicular to the plates separated by the distance $a$,

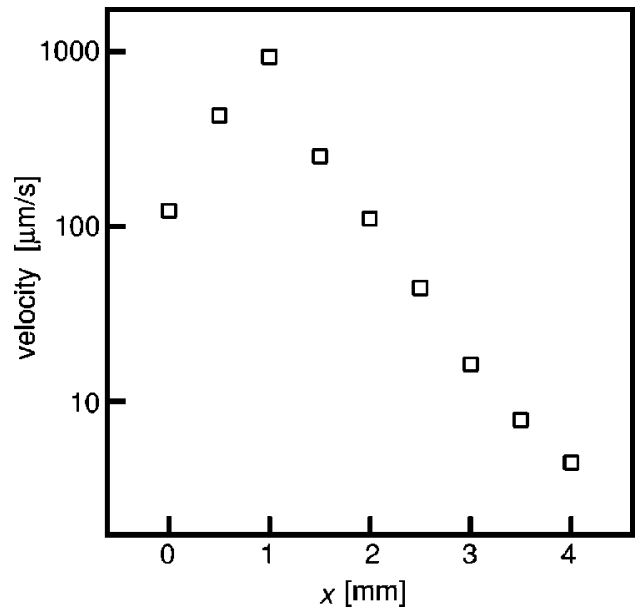

FIG. 13. Velocity along the central $x$ direction at the positions shown by squares in Fig. 12.

$$
\mathbf{F}(\mathbf{x})=\left[\mathbf{f}_{+} \delta(x-a / 2)+\mathbf{f}_{-} \delta(x+a / 2)\right] \delta(y),
$$

where $(x, y)=\mathbf{r}$ are coordinates spanning the planes parallel to the plates and $z$ is a coordinate perpendicular to the plates with $z=0$ on the lower and $z=d$ on the upper plate. The two forces $\mathbf{f}_{ \pm}$are assumed to have the same component in the $z$ direction and opposite components in the direction of the vector separating the two forces, i.e., in the $x$ direction, pushing the fluid away,

$$
\mathbf{f}_{ \pm}=f_{z} \mathbf{e}_{z} \pm f \mathbf{e}_{x}
$$

where $\mathbf{e}_{x, z}$ are the unit vectors in the $x$ and $z$ direction, respectively. This simplified dipolar force field has the same symmetry as observed in the experiment. We expect that outside the narrow spatial domain where the actual force does not vanish, this presents a fairly good approximation to the actual situation. Because of the linearity of the Stokes equations (8) and (9), it is sufficient to consider a single line-force contribution. The final result can be obtained by the superposition of the solutions to the two forces.

Having only one line force, we may put the position of coordinate center at the position of the force. Further, we note that one formally may include the $z$ component of the force into the pressure term by introducing the effective pressure

$$
p_{\text {eff }}(\mathbf{x})=\tilde{p}_{2}(\mathbf{x})-f_{z} z \delta(x) \delta(y) .
$$

Measuring lengths, velocities, and pressure in units of $d$, $f / \mu$, and $f / d$, respectively, leads to the dimensionless Stokes equations,

$$
\begin{gathered}
\Delta \mathbf{v}(\mathbf{x})-\nabla p_{\text {eff }}(\mathbf{x})=-\mathbf{e}_{x} \delta^{2}(\mathbf{r}), \\
\boldsymbol{\nabla} \cdot \mathbf{v}(\mathbf{x})=\mathbf{0},
\end{gathered}
$$

with no-slip boundary conditions on the plates,

$$
\mathbf{v}(\mathbf{r}, 0)=\mathbf{v}(\mathbf{r}, 1)=\mathbf{0},
$$

where $\delta^{2}(\mathbf{r})=\delta(x) \delta(y)$ is a two-dimensional $\delta$ function. If one takes the divergence of the Stokes equation, one finds a Poisson equation for the effective pressure,

$$
\Delta p_{\text {eff }}(\mathbf{x})=\frac{\partial}{\partial x} \delta^{2}(\mathbf{r})
$$

Because the inhomogeneity on the right-hand side is $z$-independent, it follows that the effective pressure also does not depend on $z$. Consequently, the $z$ component of the velocity fulfills the Laplace equation with homogeneous boundary conditions and therefore $v_{z}(\mathbf{x})$ vanishes everywhere. The remaining two components of the velocity field can be expressed in terms of a stream function $\Phi(\mathbf{x})$,

$$
v_{x}(\mathbf{x})=\frac{\partial \Phi(\mathbf{x})}{\partial y}, \quad v_{y}(\mathbf{x})=-\frac{\partial \Phi(\mathbf{x})}{\partial x} .
$$

Differentiating the $x$ and $y$ components of the Stokes equation with respect to $y$ and $x$, respectively, and subtracting them, one obtains for the stream function 


$$
\Delta_{\mathbf{r}} \Delta \Phi(\mathbf{x})=-\frac{\partial}{\partial y} \delta^{2}(\mathbf{r}),
$$

where $\Delta_{\mathbf{r}}=\partial^{2} / \partial x^{2}+\partial^{2} / \partial y^{2}$ denotes the two-dimensional Laplace operator in the $(x, y)$ plane. Boundary conditions for $\Phi(\mathbf{x})$ are

$$
\begin{gathered}
\Phi(\mathbf{r}, 0)=\Phi(\mathbf{r}, 1)=0, \\
\frac{\partial \Phi(\mathbf{x})}{\partial x}=\frac{\partial \Phi(\mathbf{x})}{\partial y}=0, \quad \text { for } z=0,1 \\
\lim _{r \rightarrow \infty} \Phi(\mathbf{r}, z)=0,
\end{gathered}
$$

where $r=\left(x^{2}+y^{2}\right)^{1 / 2}$ denotes the absolute value of $\mathbf{r}$. Because of the periodic boundary conditions with respect to $z$, the stream function can be represented as a sine series,

$$
\Phi(\mathbf{x})=\sum_{n=1}^{\infty} c_{n}(\mathbf{r}) \sin (\pi n z) .
$$

Here the coefficients are still functions of $\mathbf{r}$ and obey the equations

$$
\Delta_{\mathbf{r}}\left(\Delta_{\mathbf{r}}-(\pi n)^{2}\right) c_{n}(\mathbf{r})=-\gamma_{n} \frac{\partial}{\partial y} \delta(\mathbf{r})
$$

where

$$
\gamma_{n}=2 \int_{0}^{1} d z \sin (\pi n z)=\left\{\begin{array}{cc}
0 & \text { for } n \text { even } \\
\frac{4}{\pi n} & \text { for } n \text { odd } .
\end{array}\right.
$$

The solutions of these equations can be expressed in terms of the Green's functions of the time-independent twodimensional Klein-Gordon equation,

$$
\left(\Delta_{\mathbf{r}}-k^{2}\right) g_{m}(\mathbf{r})=\delta^{2}(\mathbf{r}),
$$

where $k$ is a positive number. This Green's function is given by [20]

$$
g_{k}(\mathbf{r})=-\frac{1}{2 \pi} K_{0}(k r),
$$

where $K_{\nu}(z)$ is the modified Bessel function of order $\nu$, see Ref. [21]. To achieve this goal, we introduce an auxiliary function $h(r)$ and rewrite Eq. (23) in terms of two KleinGordon equations,

$$
\begin{gathered}
\Delta_{\mathbf{r}} h(\mathbf{r})=-\frac{\partial}{\partial y} \delta^{2}(\mathbf{r}), \\
{\left[\Delta_{\mathbf{r}}-(n \pi)^{2}\right] c_{n}(\mathbf{r})=\gamma_{n} h(\mathbf{r}) .}
\end{gathered}
$$

The solution to the first equation can readily be expressed in terms of the Green's function for $m=0$,

$$
h(\mathbf{r})=-\lim _{m \rightarrow 0} \frac{\partial}{\partial y} g_{m}(\mathbf{r})=\frac{1}{2 \pi} \frac{\partial}{\partial y} \ln r .
$$

From the second equation, one then finds

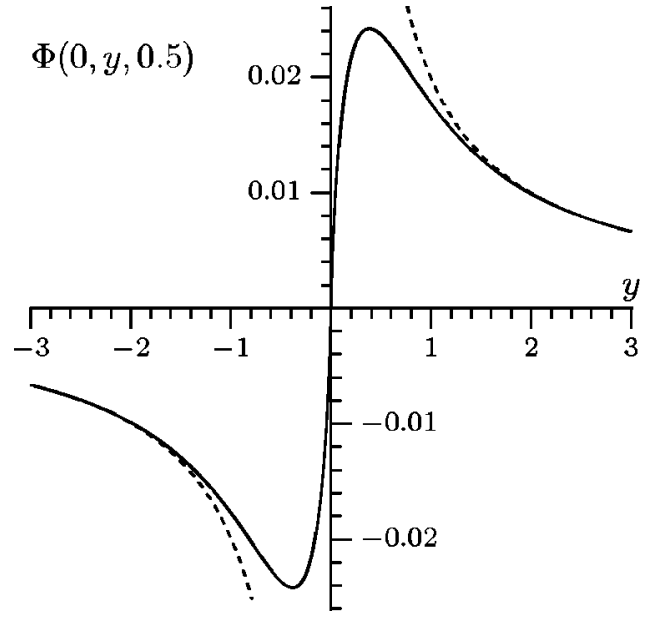

FIG. 14. The streaming function $\Phi(0, y, 0.5)$ (solid line) perpendicular to the axis $x=0$ displays extrema close to $|y|=0.3$. The broken line shows the asymptotic far field behavior according to Eq. (32). Both functions perfectly coincide for distances from the origin that are larger than twice the plate distance.

$$
\begin{aligned}
c_{n}(\mathbf{r}) & =\gamma_{n} \int d^{2} \mathbf{r}^{\prime} g_{n \pi}\left(\mathbf{r}-\mathbf{r}^{\prime}\right) h\left(\mathbf{r}^{\prime}\right) \\
& =\frac{\gamma_{n}}{(2 \pi)^{2}} \frac{\partial}{\partial y} \int d^{2} \mathbf{r}^{\prime} K_{0}\left(n \pi r^{\prime}\right) \ln \left|\mathbf{r}-\mathbf{r}^{\prime}\right| .
\end{aligned}
$$

Introducing polar coordinates, one may perform the integration over $\mathbf{r}$ and then obtains

$$
c_{n}(\mathbf{r})=\left\{\begin{array}{cl}
0 & \text { for } n \text { even } \\
\frac{2 y}{n^{3} \pi^{4} r^{2}}\left[1-n \pi r K_{1}(n \pi r)\right] & \text { for } n \text { odd } .
\end{array}\right.
$$

The modified Bessel function decays rapidly for large values of its argument and therefore $n \pi r K_{1}(n \pi r)$ can be neglected for $\pi r \gg 1$. This gives the asymptotic behavior of the stream function,

$$
\Phi_{\mathrm{as}}(\mathbf{x})=\frac{y}{4 \pi r^{2}} z(1-z),
$$

where we summed the sine series $\sum_{n \text { odd }}\left[\sin (n \pi) z / n^{3}\right]$ $=\left(\pi^{3} / 8\right) z(1-z)$, see Ref. [22]. Deviations from this asymptotic result can only be seen within a distance of a few gap widths from the location where the force is acting; see Figs. 14 and 15.

The streaming function of the force dipole readily follows as the linear combination of two velocity fields that are caused by slightly displaced line forces pointing in opposite lateral directions,

$$
\Phi_{\text {dipole }}(\mathbf{x})=\Phi(\mathbf{x}-\mathbf{e} \epsilon / 2)-\Phi(\mathbf{x}+\mathbf{e} \epsilon / 2),
$$

where $\epsilon$ is the width and $\mathbf{e}$ the unit vector in the direction of the displacement. In the following, we will assume that both the displacement and the forces show in the $x$ direction. Using the asymptotic expression (32) and taking the leading contribution with respect to the distance $\epsilon$, one obtains 


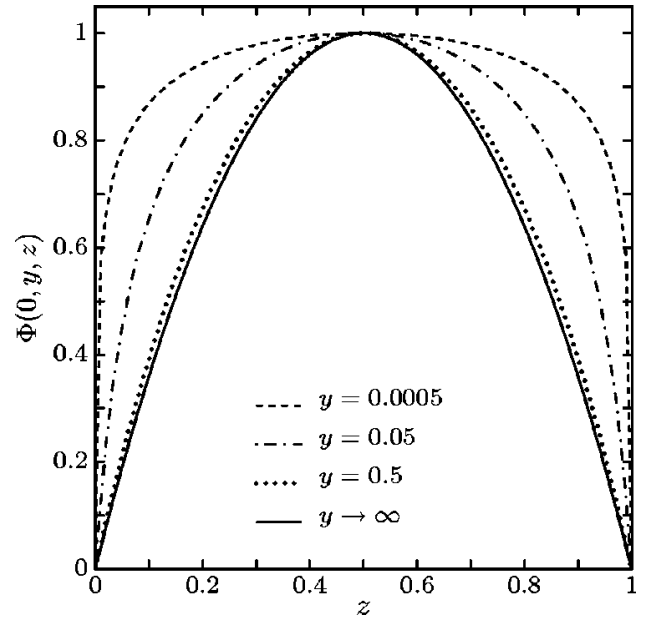

FIG. 15. The streaming function $\Phi(0, y, z) / \Phi(0, y, 0.5)$ divided by its maximal value at $z=0.5$ at $x=0$ is shown as a function of the transversal coordinate $z$ for different distances $y$ from the origin where the force is sitting. In the very close vicinity of the force source, this profile is flat in the middle part of the fluid layer. For larger distances from the force, it rapidly approaches the asymptotic parabolic profile, see Eq. (32).

$$
\Phi_{\text {dipole }}^{\text {as }}=\epsilon \frac{y x}{8 \pi r^{4}} z(1-z) .
$$

The velocity field then becomes asymptotically

$$
\begin{gathered}
v_{x}^{\mathrm{as}}(\mathbf{x})=\frac{\epsilon x}{8 \pi r^{4}}\left(1-4 \frac{y^{2}}{r^{2}}\right) z(1-z), \\
v_{y}^{\mathrm{as}}(\mathbf{x})=-\frac{\epsilon y}{8 \pi r^{4}}\left(1-4 \frac{x^{2}}{r^{2}}\right) z(1-z), \\
v_{z}(\mathbf{x})=0 .
\end{gathered}
$$

Note that the near field has a less pronounced, logarithmic singularity. The full two-dimensional vector field $v_{x}(\mathbf{r}, 0.5)$, $v_{y}(\mathbf{r}, 0.5)$ that follows from Eqs. (17), (22), (31), and (33) is shown for a pair of opposite line forces pointing in the positive and negative $x$ direction at $\mathbf{r}=( \pm 1,0)$ in Fig. 16. In this case, the fluid is sucked along the $y$ axis and pushed out in the $\pm x$ direction generating a whirl, or eddy, in each quadrant close to the dipole generating a quadrupolar velocity pattern.

\section{B. Motion of advected particles}

We consider the motion of a small particle that is advected by the flow field (35). Its trajectory agrees with the streamlines of the vector field $\mathbf{v}(\mathbf{x})$ which are the solutions of

$$
\dot{\mathbf{x}}(t)=\mathbf{v}(\mathbf{x}(t)) .
$$

Because the velocity component in the $z$ direction vanishes, the motion of a particle is strictly confined to the initial plane $z=z(0)$ as long as diffusion can be neglected. Figure 16 shows the form that an initially circular spot in the central plane $\left(z=\frac{1}{2}\right)$ acquires at four later times. We note that the area covered by the spot does not change because the vector field
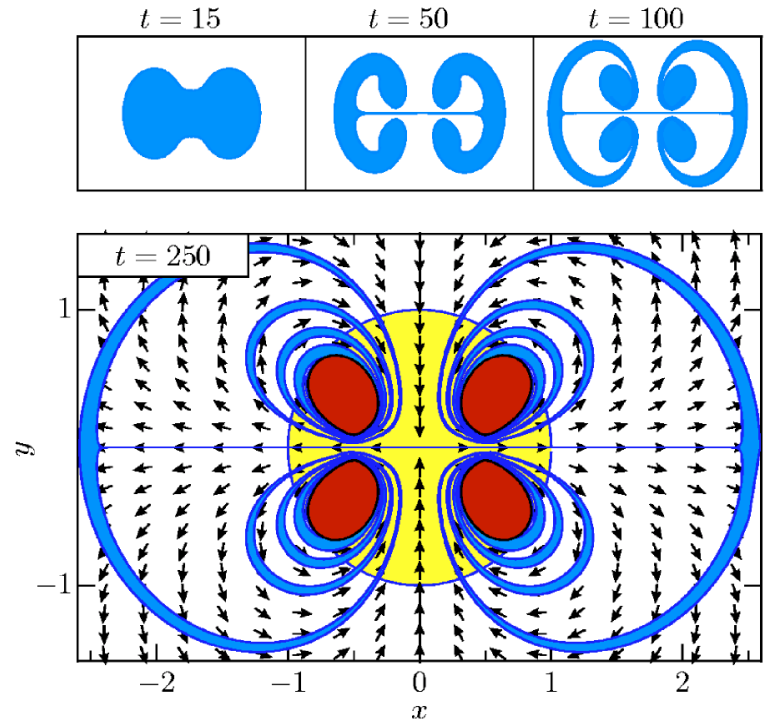

FIG. 16. (Color online) The graph shows the calculated evolution of a dye spot pumped by a SAW transducer in the center of the images. The black arrows mark the direction of the velocity field. The initial dye spot is marked by the lightly shaded circular region. The four black ellipses in the lower image represent areas where the dye is trapped by an eddy. The trajectories were calculated for the plane in the middle of the fluid film with diffusion neglected.

has vanishing divergence. The apparent change of the area of a dye spot which is observed in the experiment is due to the fact that different layers that move differently are superimposed. The alternating stripes of colored and uncolored fluid that evolve after sufficiently long time within each vertical layer cover each other and therefore cannot easily be seen in the experiment. In the course of time, these layers become ever thinner and eventually diffusion becomes effective. Around the center of each of the four eddies, regions exist that remain invariant under the flow, i.e., there is no transport out of these regions or into them other than diffusion.

Finally, we consider a particle that initially sits in the middle position between the plates on the $x$ axis at a distance $x_{0}$ from the origin. It moves with the fluid strictly in the $x$ direction according to

$$
\frac{d}{d t} x(t)=v_{x}(x(t), 0,0.5)=\frac{c}{x(t)^{3}},
$$

where $c=\epsilon / 32 \pi$ is a constant. The solution of this differential equation reads

$$
x(t)=\left(x_{0}^{4}+c t\right)^{1 / 4} .
$$

Here we have neglected the influence of diffusion. This will be of primary importance for the motion in the $z$ direction because there is no systematic force in this direction and, moreover, the $z$ coordinate of the particle position strongly influences its lateral velocity. A diffusional motion in the $z$ direction averages over $z$ and results in an effective value of the constant $c$ which becomes smaller by the factor $\frac{2}{3}$.

In order to compare with the theoretical prediction of Eq. (38), in Fig. 17 the fourth power of the position is plotted against time for four different rf-power values. Except for the 


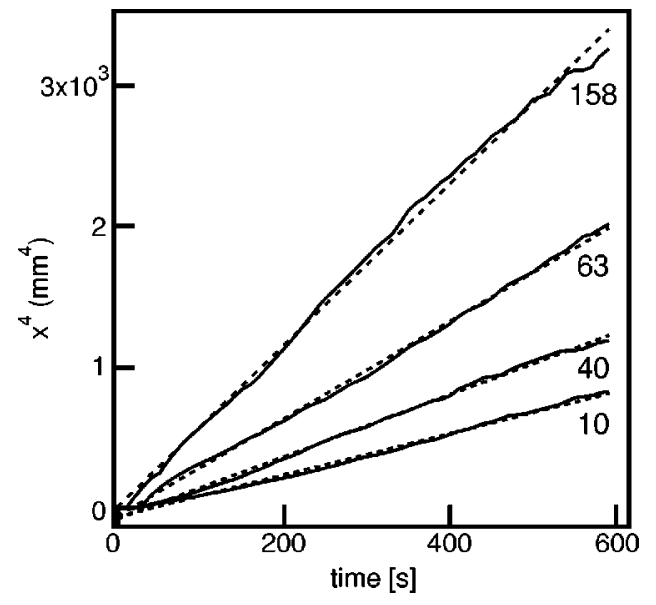

FIG. 17. The fourth power of the front positions (solid line) for four values of rf power $(10,40,63$, and $158 \mathrm{~mW}$ from bottom to top) of the dye spot indicated by the arrow in Fig. 6 measured at different instants of time (see also from Fig. 7) falls on straight lines as predicted by the asymptotic law given in Eq. (38). Only for the very first points and at high rf power do deviations appear. From the inclination of the linear fits (broken lines), one obtains a reliable fit of the constant $c$ that determines the velocity field, see Eq. (37).

highest power value where the asymptotic velocity field Eq. (37) might not be fully applicable, the experimental data nicely fall onto straight lines. From the slope of this line we obtain the constant $c$ which determines the velocity field, Eq. (37). The value of $c$ increases with the applied rf power, see Fig. 18. An extrapolation of a linear fit of the data, however, would lead to a spurious finite positive value of $c$ and consequently to finite velocities in the absence of driving. We therefore conclude that the increase of $c$ with the rf power must be weaker than linear.

\section{DISCUSSION}

We applied fluorescence microscopy and FCS for the flow profile analysis of a SAW transducer in a $200 \mu \mathrm{m}$ thin fluid

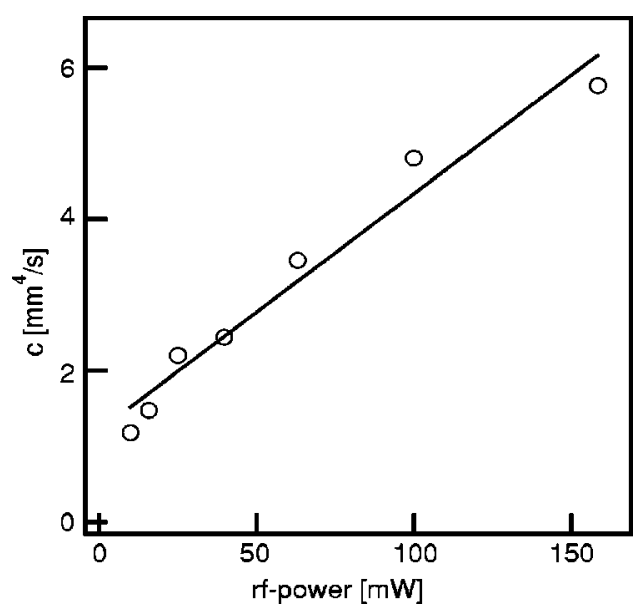

FIG. 18. The constant $c$ as determined from measurements of the position of the dye front is shown for different values of the rf power. film. With the first technique the growth of the area of a Fluorescein spot was measured. From this, the front displacement in the $x$ direction could be determined. No qualitative difference in the movement was found for different rf-power values.

In the FCS experiments, the flow speed in a homogeneous dispersion of fluorescent beads was measured at different points in a 5-mm-wide channel. A fast decay of the flow speed with distance from the transducer was found.

As a theoretical model, the Navier-Stokes equations were solved for an infinite fluid layer in the Stokes limit of low Reynolds numbers. The conversion of the sound wave into a steady flow was described in terms of body forces that were further simplified as line forces. Because the actual spatial extension of the body forces is confined to a narrow regime, this seems to be a reasonable approximation. The resulting flow field is in good qualitative agreement with the experimental findings. According to the theoretical prediction, the velocity field asymptotically decays with the third inverse power of the distance from the IDT. The resulting motion of the front of a dye spot in the symmetry direction is in quantitative agreement with the measurements.

To determine the distance at which the influence of the transducer on the dye molecules vanishes, the growth speed $\left(v_{A}\right)$ of the Fluorescein-covered area was measured. Plotting these data against the actual position of the advancing front shows a fast decay until the diffusion of the dye sets in. This distance depends logarithmically on the rf power and is about $8 \mathrm{~mm}$ for the maximum applied $158 \mathrm{~mW}$ in the microscope study.

We measured the flow speed at different points on the streamlines and found a linear dependence of the speed on the rf power. However, when the constant $c$ in Eq. (38) is determined from the time dependence of the measured front positions for different hf values, the data cannot be fitted satisfactorily by a straight line through the origin. Following Eq. (37), the velocity at a fixed point in the fluid cannot depend strictly linearly on the power. Yet in the experimentally restricted regime of space and power, a linear law gives a reasonable fit.

The two applied methods provide complementary aspects of the fluid motion in the thin film. FCS is the more quantitative method that yields exact values of the flow speed of particles at any position in the film. Only for very high and rather low speeds does one have to deal with the limitations of the method. In particular, the transition area from advected to diffusive transport cannot be located correctly. With the microscope method, this is feasible with the evaluation of the determined area growth speed. The time-dependent displacement data gained with this method can further be used to compare with the theoretical predictions.

Finally, we address the question of what one can learn from the presented experiments and theory in order to accelerate the hybridization of DNA in a thin fluid film with the lateral size of a normal glass slide $(75 \times 25 \mathrm{~mm})$. For this size, diffusive transport becomes extremely slow and should be complemented by advected transport. The flow induced by surface acoustic waves can be efficiently used for this purpose. However, because of the rapid decay of the induced velocity field away from the transducer, the maximal mixing 
range was found to have a linear dimension of about $8 \mathrm{~mm}$ in the present experiment at the highest rf power. A further increase of the power does not seem feasible because it would enlarge the mixing range only insignificantly. To account for the limited range of the induced flow by the SAW transducer, the number of transducers on a slide should be increased. In the calculated flow field in Fig. 16 four eddies appear close to the transducer where particles can be trapped. To avoid this problem, pairs of transducers should always be operated alternately. So the trapped particles from one transducer will be moved by the other and vice versa.

\section{ACKNOWLEDGMENTS}

The authors gratefully acknowledge financial support by the Deutsche Forschungsgemeinschaft (DFG) under the Sonderforschungsbereich 486 and 563 and the Bayerische Forschungsstiftung under the Programme "ForNano."
[1] A. Kamholz and P. Yager, Biophys. J. 80, 155 (2001).

[2] N. Darnton, O. Bakajin, R. Huang, B. North, J. Tegenfeldt, E. C. Cox, J. Sturm, and R. Austin, J. Phys.: Condens. Matter 13, 4891 (2001).

[3] J. H. Wu and H. J. Keh, Colloids Surf., A 212, 27 (2003).

[4] S. Zeng, C. H. Chen, J. C. Mikkelsen, and J. G. Santiago, Sens. Actuators B 79, 107 (2001).

[5] S. Maiti, U. Haupts, and W. W. Webb, Proc. Natl. Acad. Sci. U.S.A. 94, 11753 (1997).

[6] S. Shiokawa, Y. Matsui, and T. Ueda, Proc.-IEEE Ultrason. Symp. 1, 643 (1989).

[7] J. Campbell and W. Jones, IEEE Trans. Sonics Ultrason. 17, 71 (1970)

[8] W. Nyborg, Phys. Acoust. 2B, 265 (1965).

[9] T. Uchida, T. Suzuki, and S. Shiokawa, Proc.-IEEE Ultrason. Symp. 2, 1081 (1995).

[10] D. Magde, E. Elson, and W. W. Webb, Phys. Rev. Lett. 29, 705 (1972)

[11] P. Schwille, J. Bieschke, and F. Oehlenschlager, Biophys. Chem. 66, 211 (1997).

[12] O. Krichevsky and G. Bonnet, Rep. Prog. Phys. 65, 251
(2002).

[13] N. L. Thompson, A. M. Lieto, and N. W. Allen, Curr. Opin. Struct. Biol. 12, 634 (2002).

[14] S. T. Hess and W. W. Webb, Biophys. J. 83, 2300 (2002).

[15] M. Gösch, H. Blom, T. Heino, and R. Riegler, Anal. Chem. 72, 3260 (2000).

[16] M. Eigen and R. Rigler, Proc. Natl. Acad. Sci. U.S.A. 91, 5740 (1994).

[17] K. Starchev, J. W. Zhang, and J. Buffle, J. Colloid Interface Sci. 203, 189 (1998).

[18] K. Starchev, J. Buffle, and E. Perez, J. Colloid Interface Sci. 213, 479 (1999).

[19] J. P. Brody, P. Yager, R. E. Goldstein, and R. H. Austin, Biophys. J. 71, 3430 (1996).

[20] S. Albeverio, F. Gesztesy, R. Høegh-Krohn, and H. Holden, Solvable Models in Quantum Mechanics (Springer-Verlag, Berlin, 1988).

[21] M. Abramowitz and I. Stegun, Handbook of Mathematical Functions (Dover Publications, New York, 1972).

[22] I. N. Bronsthein and K. A. Semendyayev, Handbook of Mathematics (van Nostrand Reinhold Company, New York, 1985). 